\title{
The $X_{c}(3250)$ as a $D^{*} \Delta$ molecule
}

\author{
P.G. Ortega \\ CERN (European Organization for Nuclear Research), CH-1211 Geneva, Switzerland \\ E-mail: pablo.garcia.ortega@cern.ch

\section{D.R. Entem*} \\ Departamento de Física Fundamental and IUFFyM, University of Salamanca, E-37008 \\ Salamanca, Spain \\ E-mail: entemeusal.es

\section{F. Fernández} \\ Departamento de Física Fundamental and IUFFyM, University of Salamanca, E-37008 \\ Salamanca, Spain \\ E-mail: fdzeusal.es
}

A new structure at $3.25 \mathrm{GeV} / \mathrm{c}^{2}$ has been recently reported by the BaBar collaboration which has received the name of $X_{c}(3250)$. A preliminary fit to this structure gives a mass $\mathrm{M}=3245 \pm$ $20 \mathrm{MeV} / \mathrm{c}^{2}$ and a width $\Gamma=108 \pm 60 \mathrm{MeV} / \mathrm{c}^{2}$. A molecular interpretation as a $D_{0}^{*}(2400) N$ bound state has been proposed since the threshold for this channel is close to the nominal mass. However the width of the $D_{0}^{*}(2400)$ seems to be too large to explain the width of the $X_{c}(3250)$. As the $D^{*} \Delta$ threshold is also close to the nominal mass of the state, we investigate the possibility to be a $D^{*} \Delta$ bound state. We find three possible molecules with $I\left(J^{P}\right)$ quantum numbers $2\left(\frac{1}{2}^{-}\right), 2\left(\frac{3}{2}^{-}\right)$and $1\left(\frac{5}{2}^{-}\right)$which are compatible with the measured mass and width.

XV International Conference on Hadron Spectroscopy-Hadron 2013

4-8 November 2013

Nara, Japan

${ }^{*}$ Speaker. 


\section{Introduction}

A new structure at $3.25 \mathrm{GeV} / c^{2}$ has been recently reported by the BaBar collaboration in the $\Sigma_{c}^{++} \pi^{-} \pi^{-}$invariant mass spectrum [1] which has received the name of $X_{c}(3250)$. A preliminary Breit-Wigner plus background fit to this structure gives a mass $M=3245 \pm 20 \mathrm{MeV} / c^{2}$ and a width $\Gamma=108 \pm 60 \mathrm{MeV} / c^{2}$ [2].

Soon after the experimental observation He et al. [3] suggested that the $X_{c}(3250)$ could be a $D_{0}^{*}(2400) N$ molecular state. This hypothesis has been tested in a QCD sum rule calculation by Zhang [ [†. The conclusion of this work is that the conventional OPE convergence should be released to obtain a state with a mass of $3.18 \mathrm{GeV} / \mathrm{c}^{2}$. Therefore only weak conclusions can be drawn regarding the explanation of the $X_{c}(3250)$ as a $D_{0}^{*}(2400) N$ molecular state in this framework. One reason to suspect why this description may fail is that the $D_{0}^{*}(2400)$ resonance is too broad $(\Gamma=267 \mathrm{MeV})$ which makes difficult to justify an experimental width of the order of $100 \mathrm{MeV}$.

In this work we propose an alternative description of the $X_{c}(3250)$ as a $D^{*} \Delta$ molecule. In this case, the threshold is located at $3240 \mathrm{MeV} / c^{2}$ so the $X_{c}(3250)$ state is almost at threshold. Furthermore the width of the $\Delta$ fits better with the experimental result.

We will use a similar framework that has been used to study many different hadron molecules. One typical example is the $X(3872)$ which can decay into $J / \psi \rho$ and so completely rules out a $c \bar{c}$ interpretation. In Ref. [5] we performed a study of this state as a possible $D D^{*}$ molecule coupled to $c \bar{c}$ states within the framework of the CQM of Ref. [6], finding an overall good description of some measured properties. Other $X Y Z$ states have been studied in Ref. [П]. This model also describe the deuteron as a $N N$ bound state[8] and predicts a $\Delta \Delta$ bound state[9] with the quantum numbers proposed by the WASA-at-COSY collaboration[10].

In the baryon sector the $\Lambda_{c}(2940)$ has been proposed as a possible $D^{*} N$ molecule [11] within the same model.

\section{The Chiral Quark Model}

We will use the CQM of Refs. [6, 12]. In these references all the details of the model can be found and here we only summarize the most important aspects.

One of the most important features of QCD at low energies is the spontaneous breaking of chiral symmetry. The model uses the most simple Lagrangian that describes this effect which can be written as

$$
\mathscr{M}=\bar{\Psi}\left(i \gamma^{\mu} \partial_{\mu}-M U^{\gamma_{5}}\right) \Psi
$$

where $U^{\gamma_{5}}=e^{i \pi^{a} \lambda^{a} \gamma_{5} / f_{\pi}} \sim 1+\frac{1}{f_{\pi}} \gamma_{5} \lambda^{a} \pi^{a}-\frac{1}{2 f_{\pi}^{2}} \pi^{a} \pi^{a}$ with $\pi^{a}$ the pseudo-Goldstone meson octet. This effect generates the constituent quark mass for light quarks and the interaction between quarks through Goldstone bosons.

Another crucial non-perturbative effect of low energy QCD is confinement. This fact prevents hadrons to be in colored states and we used a linear screened confinement.

QCD perturbative effects are included with the coupling between quarks and gluons given by

$$
\mathscr{L}_{g q q}=i \sqrt{4 \pi \alpha_{s}} \bar{\Psi} \gamma_{\mu} G_{c}^{\mu} \lambda^{c} \Psi
$$

which gives rise to the one gluon exchange interaction. 


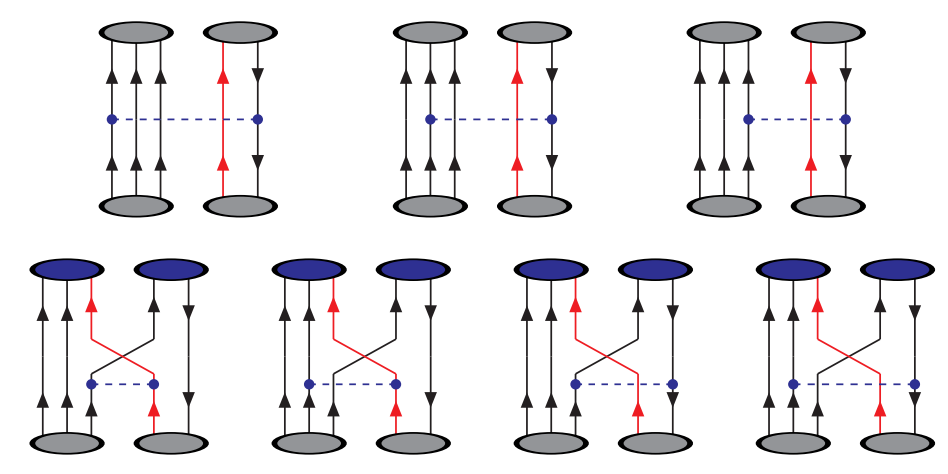

Figure 1: Diagrams that contribute to the meson-baryon interaction (first row) and to rearrangement processes (second row).

\begin{tabular}{cccccc}
\hline \hline$J^{P}$ & $I$ & state & Mass $\left(\mathrm{MeV} / \mathrm{c}^{2}\right)$ & $E_{b}(\mathrm{MeV})$ & $P_{\max }($ Channel $)$ \\
\hline \hline$\frac{1}{2}^{-}$ & 2 & $D^{*} \Delta$ & 3233 & -6.47 & $99.71\left({ }^{2} S_{1 / 2}\right)$ \\
$\frac{3}{2}^{-}$ & 1 & $D \Delta$ & 3097 & -0.88 & $99.13\left({ }^{4} S_{3 / 2}\right)$ \\
$\frac{3}{2}^{-}$ & 2 & $D^{*} \Delta$ & 3238 & -0.98 & $99.69\left({ }^{2} S_{1 / 2}\right)$ \\
$\frac{5}{2}^{-}$ & 1 & $D^{*} \Delta$ & 3226 & -13.12 & $97.25\left({ }^{6} S_{5 / 2}\right)$ \\
\hline \hline
\end{tabular}

Table 1: Possible $D^{(*)} \Delta$ states found within the model. The mass, binding energy and maximum partial wave probability are given.

\section{The meson-baryon interaction}

The meson-baryon interaction is obtained using the Resonating Group Method. No antisymmetry effects are present and the interaction is given by

$$
V_{D}=\sum_{i \in A ; j \in B} \int \Psi_{\alpha_{A^{\prime}}}^{\dagger}\left(\vec{p}_{A}^{\prime}\right) \Psi_{\alpha_{B^{\prime}}}^{\dagger}\left(\vec{p}_{B}^{\prime}\right) V_{i j}\left(\vec{P}^{\prime}, \vec{P}\right) \Psi_{\alpha_{A}}\left(\vec{p}_{A}\right) \Psi_{\alpha_{B}}\left(\vec{p}_{B}\right)
$$

where $V_{D}$ is the Direct RGM kernel and $\Psi_{\alpha_{C}}\left(\vec{p}_{C}\right)$ are the internal wave functions of a $C$ baryon or heavy meson with $\alpha_{C}$ quantum numbers. The diagrams contributing to $V_{D}$ are shown in the first row of Fig.11.

However processes in which a quark is exchanged between the meson and the nucleon (rearrangement processes) are possible and give rise to different decay modes. The contributing diagrams are represented in the second row of Fig.1.

\section{Results}

First we have performed a calculation looking for possible $D_{0}^{*}(2400) N$ bound state and we have not found any in the sectors with quantum numbers $J^{P}\left(\frac{1}{2}^{+}\right),\left(\frac{1}{2}^{-}\right)$and $\left(\frac{3}{2}^{-}\right)$. We then calculated possible $D^{(*)} \Delta$ molecular states and their bottom partners. The results are summarized in Tables 11 and 2 . In the charm sector we find one $D \Delta$ state and three $D^{*} \Delta$, being one of these last three states our candidate for the $X_{c}(3250)$. All of them have a bottom partner and we find one additional state in the $\bar{B} \Delta$ and $\bar{B}^{*} \Delta$ sectors. 


\begin{tabular}{cccccc}
\hline \hline$J^{P}$ & $I$ & state & Mass $\left(\mathrm{MeV} / \mathrm{c}^{2}\right)$ & $E_{b}(\mathrm{MeV})$ & $P_{\max }($ Channel $)$ \\
\hline \hline$\frac{1}{2}^{-}$ & 2 & $\bar{B}^{*} \Delta$ & 6541 & -14.21 & $99.69\left({ }^{2} S_{1 / 2}\right)$ \\
$\frac{3}{2}^{-}$ & 1 & $\bar{B} \Delta$ & 6499 & -10.72 & $88.14\left({ }^{4} S_{3 / 2}\right)$ \\
$\frac{3}{2}^{-}$ & 2 & $\bar{B} \Delta$ & 6506 & -3.67 & $94.72\left({ }^{4} S_{3 / 2}\right)$ \\
$\frac{3}{2}^{-}$ & 1 & $\bar{B}^{*} \Delta$ & 6555 & -0.39 & $97.10\left({ }^{4} S_{3 / 2}\right)$ \\
$\frac{3}{2}^{-}$ & 2 & $\bar{B}^{*} \Delta$ & 6550 & -4.85 & $99.48\left({ }^{4} S_{3 / 2}\right)$ \\
$\frac{5}{2}^{-}$ & 1 & $\bar{B}^{*} \Delta$ & 6532 & -23.16 & $96.76\left({ }^{6} S_{5 / 2}\right)$ \\
\hline \hline
\end{tabular}

Table 2: Possible $\bar{B}^{(*)} \Delta$ states found within the model. The mass, binding energy and maximum partial wave probability are given.

\begin{tabular}{ccccccccc}
\hline \hline$J^{P}$ & $I$ & & $\Gamma_{D \Delta}$ & $\Gamma_{D^{*} N}$ & $\Gamma_{D N}$ & $\Gamma_{D \pi \Delta}$ & $\Gamma_{D^{*} N \pi}$ & $\Gamma_{D N \pi}$ \\
\hline \hline$\frac{1}{2}^{-}$ & 2 & $D^{*} \Delta$ & 0.005 & 0 & 0 & 0 & 111 & 0 \\
$\frac{3}{2}^{-}$ & 1 & $D \Delta$ & 0 & 1.31 & 0.001 & 0 & 0.049 & 113 \\
$\frac{3}{2}^{-}$ & 2 & $D^{*} \Delta$ & 6.18 & 0 & 0 & 0.038 & 114 & 0 \\
$\frac{5}{2}^{-}$ & 1 & $D^{*} \Delta$ & 0.003 & 1.23 & 0.64 & 0 & 108 & 0 \\
\hline \hline
\end{tabular}

Table 3: Widths (in MeV) for different decay channels of the possible $D^{(*)} \Delta$ states found.

\begin{tabular}{ccccccccc}
\hline \hline$J^{P}$ & $I$ & & $\Gamma_{\bar{B} \Delta}$ & $\Gamma_{\bar{B}^{*} N}$ & $\Gamma_{\bar{B} N}$ & $\Gamma_{\bar{B} \pi \Delta}$ & $\Gamma_{\bar{B}^{*} N \pi}$ & $\Gamma_{\bar{B} N \pi}$ \\
\hline \hline$\frac{1}{2}^{-}$ & 2 & $\bar{B}^{*} \Delta$ & 0.021 & 0 & 0 & 0 & 111 & 0 \\
$\frac{3}{2}^{-}$ & 1 & $\bar{B} \Delta$ & 0 & 3.91 & 0.02 & 0 & 0 & 98 \\
$\frac{3}{2}^{-}$ & 2 & $\bar{B} \Delta$ & 0 & 0 & 0 & 0 & 0 & 108 \\
$\frac{3}{2}^{-}$ & 1 & $\bar{B}^{*} \Delta$ & 12.47 & 0.224 & 0.019 & 0.076 & 115 & 0 \\
$\frac{3}{2}^{-}$ & 2 & $\bar{B}^{*} \Delta$ & 19.84 & 0 & 0 & 0 & 114 & 0 \\
$\frac{5}{2}^{-}$ & 1 & $\bar{B}^{*} \Delta$ & 0.001 & 0 & 0.90 & 0 & 108 & 0 \\
\hline \hline
\end{tabular}

Table 4: Widths (in MeV) for different decay channels of the possible $\bar{B}^{(*)} \Delta$ states found.

We have also analyzed different decay channels of the states found. Those are given in Tables 3 and $\emptyset$. As seen in the tables the width of the states is dominated by the $\Delta$ decay into $N \pi$, been the main decay channel for $D^{(*)} \Delta\left(\bar{B}^{(*)} \Delta\right)$ the $D^{(*)} N \pi\left(\bar{B}^{(*)} N \pi\right)$ channel.

As seen in Table 1 the $\Delta D^{*}$ hypothesis implies a negative parity of the state against a positive parity in the $D^{*}(2400) N$ hypothesis. In the second case $I=1$ is implied while in the first case $I=2$ is also possible. The main decay channel would be $D^{*} N \pi$ in the first case and $D N \pi$ in the second case. These differences will allow to distinguish between the two hypothesis. Also in both cases a bottom partner should appear, however in the first case the mass would be around $100 \mathrm{MeV} / \mathrm{c}^{2}$ lower that in the second case and will have a value in the order of $6.5 \mathrm{GeV} / \mathrm{c}^{2}$.

\section{Acknowledgments}

This work has been partially funded by Ministerio de Ciencia y Tecnología under Contract No. FPA2010-21750-C02-02, by the European Community-Research Infrastructure Integrating 
Activity 'Study of Strongly Interacting Matter' (HadronPhysics3 Grant No. 283286), the Spanish Ingenio-Consolider 2010 Program CPAN (CSD2007-00042).

\section{References}

[1] J. P. Lees et al. (BaBar collaboration), Study of the baryonic B decay $B^{-} \rightarrow \Sigma_{c}^{++} \bar{p} \pi^{-} \pi^{-}$, Phys. Rev. D 86 (2012) 091102.

[2] O. Gruenberg (BaBar collaboration), Moriond 2012, QCD and High Energy Interactions: Baryonic B decays at BaBar, Proceedings Rencontres de Moriond QCD 2012 (electronic version), arXiv:1211.0212 [hep-ex].

[3] J. He, D.-Y. Chen and X. Liu, New structure around $3250 \mathrm{MeV}$ in the baryonic B decay and the $D_{0}^{*}(2400) N$ molecular hadron, Eur. Phys. J. C 72 (2012) 2121.

[4] J.-R. Zhang, Study of $X_{c}(3250)$ as a $D_{0}^{*}(2400) N$ molecular state, Phys. Rev. D 87 (2013) 076008.

[5] P.G. Ortega, J. Segovia, D.R. Entem and F. Fernandez, Coupled channel approach to the structure of the X(3872), Phys. Rev. D 81 (2010) 054023.

[6] F. Fernandez, A. Valcarce, U. Straub and A. Faessler, The Nucleon-nucleon interaction in terms of quark degrees of freedom, Jour. Phys. G 19 (1993) 2013.

[7] P.G. Ortega, D.R. Entem and F. Fernandez, Molecular structures in the charmonium spectrum: the XYZ puzzle, Jour. Phys. G 40 (2013) 065107.

[8] D.R. Entem, F. Fernández and A. Valcarce, Chiral quark model of the $N$ N system within a Lippmann-Schwinger resonating group method, Phys. Rev. C 62 (2000) 034002.

[9] A. Valcarce, H. Garcilazo, R.D. Mota and F Fernández, $\Delta \Delta$ and $\Delta \Delta \Delta$ bound states, Jour. Phys. G 27 (2001) L1.

[10] P. Adlarsoni et al., Abashian-Booth-Crowe Effect in Basic Double-Pionic Fusion: A New Resonance?, Phys. Rev. Lett. 106 (2011) 242302.

[11] P.G. Ortega, D.R. Entem and F. Fernandez, Quark model description of the $\Lambda_{c}(2940)$ as a molecular $D^{*} N$ state and the possible existence of the $\Lambda_{b}(6248)$, Phys. Lett. B 718 (2013) 1381.

[12] J. Vijande, F. Fernandez and A. Valcarce, Constituent quark model study of the meson spectra, Jour. Phys. G 31 (2005) 481. 